\title{
Investigation of aerodynamic instability of a thin plate
}

\author{
Irina Lantsova ${ }^{{ }^{*}}$ and Irina Afanasyeva ${ }^{1,2}$ \\ ${ }^{1}$ Moscow state university of civil engineering, Yaroslavskoye shosse, 26, Moscow, Russia, 129337 \\ ${ }^{2}$ Research \& Educational Center StaDyO Ltd, Office 810, 18, 3-ya Ulitsa Yamskogo Polya, Moscow, \\ 125040 , Russia
}

\begin{abstract}
This paper is devoted to methods and principles of investigation of aerodynamic instability of structures. Object of interest is a thin plate. This is an important aspect of the design of large-span structures, bridges and other structures sensitive to wind loads. As Russian standards and Eurocode obliges to check the conditions for the occurrence of galloping, divergence and flutter for a certain class of structures. Modern computing facilities allow to calculate aerodynamic coefficients with high accuracy.
\end{abstract}

\section{Introduction}

The relevance of this work is determined by the need to take into account the sensitivity of structures to wind loads that produces aeroelastic vibrations, which can lead to collapse of the structure. This type of structures includes the large-span structures, large-span bridges, thin-walled cooling towers, high-rise buildings, roofs of long-span stadiums and so on.

Exciting analytical, experimental and numerical techniques that allow to assess the possibility of the occurrence of such aerodynamic instability effects as vortex shedding, galloping or divergence need to be improved $[1,2]$. Nowadays the modern numerical methods and computational tools let to perform detailed simulations of the aeroelastic behavior of structures and fluid flow around it, but it is necessary to verify and validate them.

\section{Problem statement}

The object of interest is a thin plate (fig. 1) placed in a viscous incompressible turbulent flow $\left(R e=7 \cdot 10^{5}\right)$. The purpose of this study is an identification of the occurrence of such aerodynamic instability effects for the interesting object, such as vortex shedding, galloping or divergence. We consider two-dimensional problem (at the plane OXY). Therefore, in the third dimension (along the axis OZ) the domain has one element and size equal $0.1 \mathrm{~m}$. For simulation of the turbulent flow RANS/URANS SST turbulence models are used (for steady state and unsteady simulations respectively). Simulation time for unsteady simulations is $40 \mathrm{~s}$ with Time step equal $0.005 \mathrm{~s}$.

* Corresponding author: irina-lanzova@mail.ru 


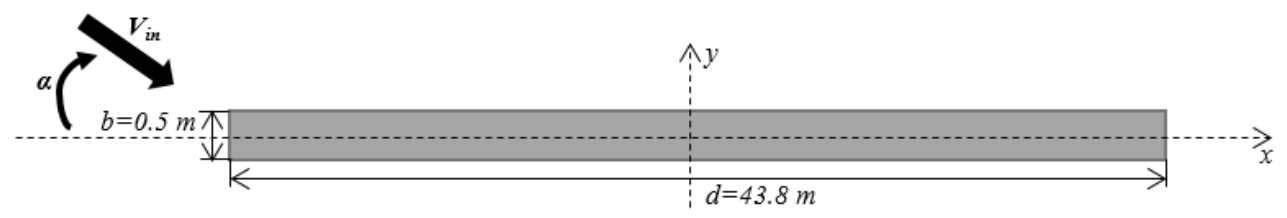

Fig. 1. Geometrical parameters of the model cross-section. $\alpha$ - angle of wind attack.

Following boundary conditions are set (Fig. 2):

- velocity at the domain inlet is constant and equal $21.7 \mathrm{~m} / \mathrm{s}$, turbulence kinetic energy $42.9 \mathrm{~m}^{2} / \mathrm{s}^{2}$ and turbulence eddy dissipation $0.94 \mathrm{~m}^{2} / \mathrm{s}^{3}$;

- «Opening» boundary conditions are defined at domain outlet with zero average relevance pressure, turbulence kinetic energy equal $42.9 \mathrm{~m}^{2} / \mathrm{s}^{2}$ and turbulence eddy dissipation equal $0.94 \mathrm{~m}^{2} / \mathrm{s}^{3}$;

- «No slip wall» boundary conditions on the surface of the plate are set;

- «Symmetry» boundary conditions are set at the left and right side of the domain.

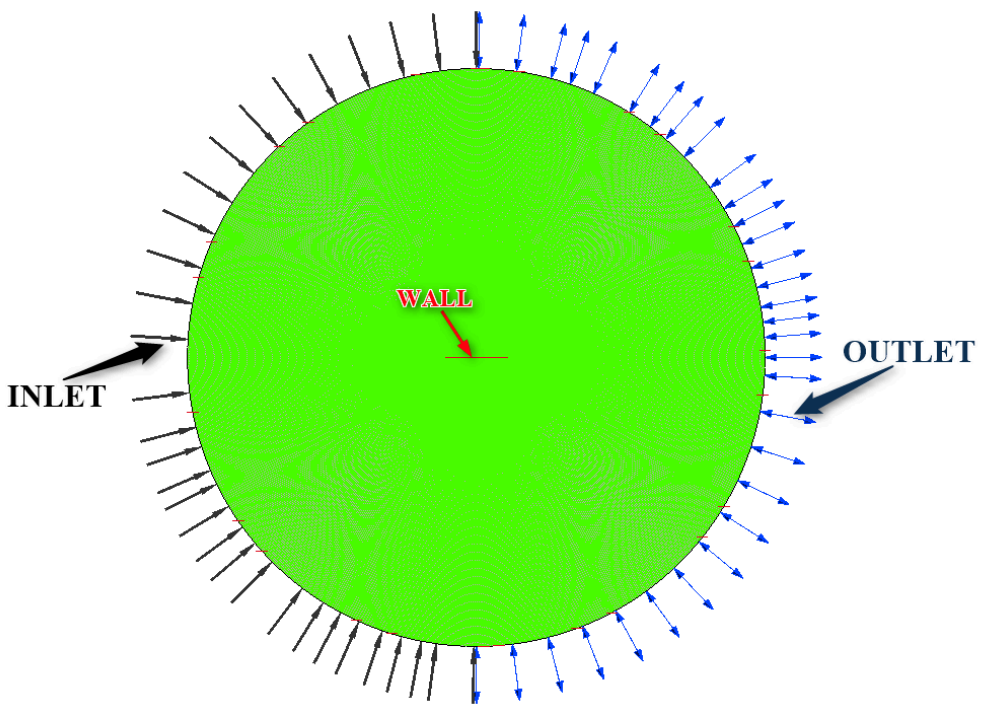

Fig. 2. Computational domain and boundary conditions.

\section{Results}

Computational domain was meshed by ANSYS Mechanical preprocessor. We considered 5 different computational grids (table 1). As controlled parameters following characteristics are chosen: aerodynamic coefficients of the drag $(C d)$ and the lift $(C l)$ forces. Model 2 (fig. 3) was chosen as a basic model for steady state simulations, Model4 (fig. 3) was chosen as a basic model for unsteady simulations. 
Table 1. Variants of the computational grids and the corresponding values of the aerodynamic coefficients of the drag $(C d)$ and the lift $(C l)$ forces. Angle of the wind attack $\alpha=1^{\circ}$.

\begin{tabular}{|c|c|c|c|c|}
\hline Name & $\begin{array}{c}\text { Number of } \\
\text { nodes }\end{array}$ & Y+ & Cd & Cl \\
\hline Model 1 & 745294 & $\sim 2500$ & 2.100 & -0.069 \\
\hline Model 2 & 1014764 & $\sim 1300$ & 2.045 & -0.070 \\
\hline Model 3 & 1676424 & $\sim 700$ & 2.017 & -0.071 \\
\hline Model 4 & 1827780 & $\sim 100$ & 2.000 & -0.071 \\
\hline Model 5 & 338092 & $\sim 43000$ & 2.257 & -0.064 \\
\hline $\begin{array}{c}\text { Ref. [3]: } \\
\text { - plate with a square cross-section }\end{array}$ & - & & 2.03 & 0 \\
- infinite thin plate with a rectangular cross-section & & - & 2.12 & - \\
\hline
\end{tabular}

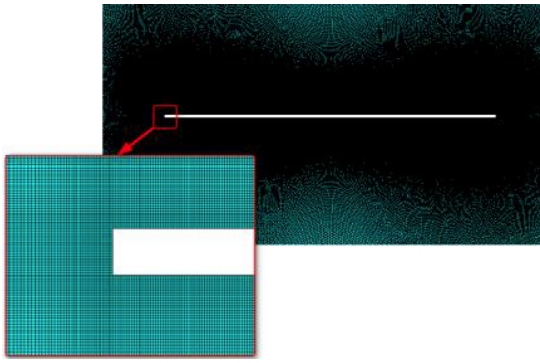

(a)

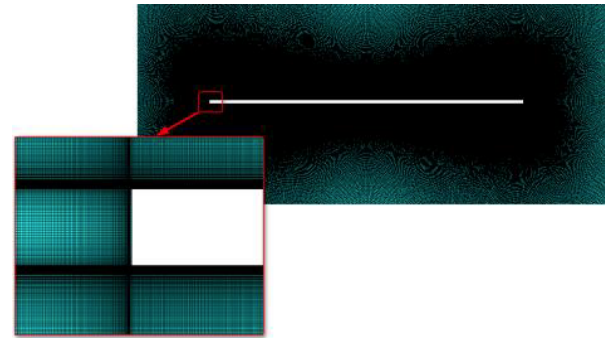

(b)

Fig. 3. Computational grid. (a) Model 2 (1 014764 nodes) and (b) Model 4 (1 827780 nodes).

In Ref. [3] the author shows the dependence of the aerodynamic coefficients of the drag $(C d)$ and the lift $(C l)$ forces on angle of attack $\alpha$ for the infinite thin plate with a rectangular cross-section. Graphs of the dependence of the obtained aerodynamic coefficients on the angle of attack $\alpha$ in comparison with the coefficients from [3] are presented below.

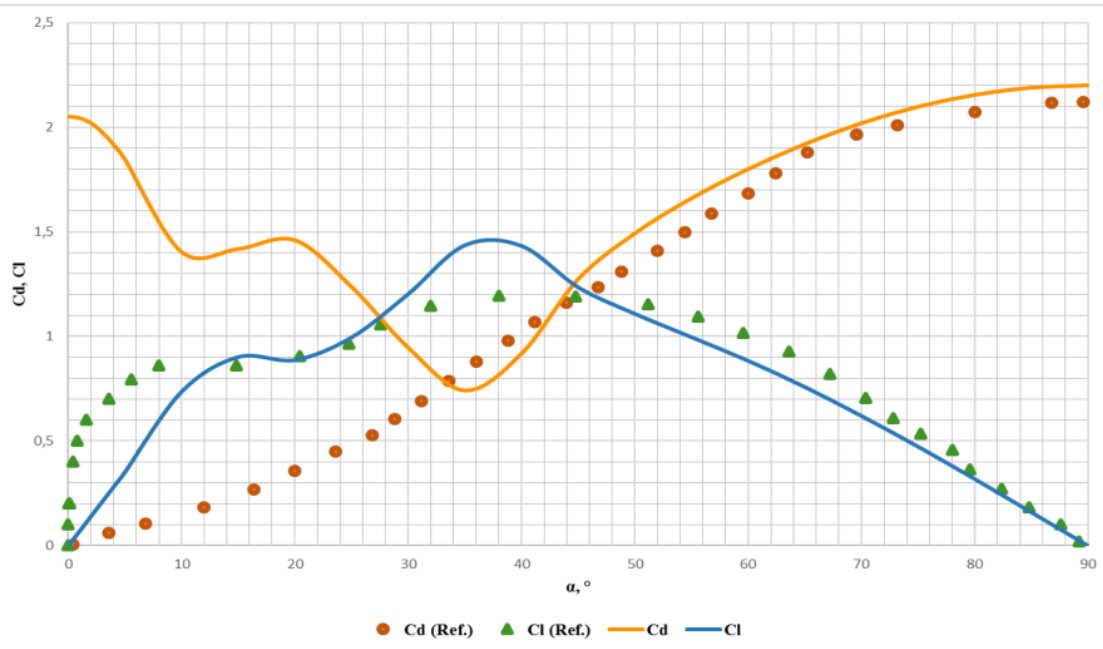

Fig. 3. Aerodynamic coefficients of the drag $(C d)$ and the lift $(C l)$ forces depending on the angle of attack $\alpha$. Comparison of the results of the simulations (Model 2) with the reference [3]. 


\subsection{Estimation of galloping, vortex shedding and divergence}

\subsubsection{Galloping}

Galloping is a self-excited oscillations of flexible structures in the form of bending vibrations along the normal to the direction of the wind

As a result of researches by scientists such as Den-Hartog and Glowrt, the necessary condition for the occurrence of an aerodynamic instability was obtained:

$$
H=C_{D}+\frac{d C_{L}}{d \alpha}<0
$$

According to the Eurocode [4], aerodynamic instability with galloping occurs when the wind speed $V_{m}$ reaches a value $V_{c G}$ at which the oscillating process begins with an increasing amplitude.

$$
V_{C G}>1.25 \cdot V_{m}
$$

The critical wind velocity of galloping, $V_{C G}$, is given in Expression (3):

$$
V_{C G}=\frac{2 \cdot S c \cdot f_{i} \cdot b}{a_{g}}
$$

where:

$f_{i}$ - cross-wind fundamental frequency of the structure;

$\mathrm{b}$ - the width;

$a_{g}$ - factor of galloping instability;

Sc - Scruton number:

$$
S c=\frac{2 \cdot \delta \cdot m}{\rho \cdot b^{2}}
$$

where:

$\delta$ - structural damping expressed by the logarithmic decrement;

$\rho$ - air density under vortex shedding conditions;

$\mathrm{m}$ - equivalent mass;

$\mathrm{b}$ - reference width of the cross-section at which resonant vortex shedding occurs.

Values of the aerodynamic coefficients of the drag $(C d)$ and the lift $(C l)$ forces at various angles of attack were determined by performing steady state simulations using specialized software ANSYS CFX. Then, according to formula (1), the Glowrt - DenGartog criterion $H$ was calculated, and the critical velocity of the beginning of the oscillatory process $V_{C G}$ was determined using formula (3) (at $f 1=0.4 \mathrm{~Hz}, S c=551$; $a g=$ $k s \cdot H$, where $k s=0.9)$. For all angles, the critical velocity exceeded the value of $1.25 \cdot V_{m}=27.1 \mathrm{~m} / \mathrm{s}$, despite the fact that for some angles of attack the Glowrt - DenGartog criterion $H$ is fulfilled (i.e. there is a possibility of the occurrence of the galoping). In the table 2, the angels of attack for which the Glowrt - Den-Gartog criterion $H$ is fulfilled are marked in red. 
Table 2. Aerodynamic coefficients of the drag $(C d)$ and the lift $(C l)$ forces, the Glowrt - Den-Gartog criterion $H$, the critical wind velocity $V_{C G}$ on angle of the wind attack $\alpha$.

\begin{tabular}{|c|c|c|c|c|}
\hline Angle $\boldsymbol{\alpha}{ }^{\circ}$ & $\mathbf{C d}$ & $\mathbf{C l}$ & $\mathbf{H}$ & $V_{C G}, \mathbf{~ m} / \mathbf{s}$ \\
\hline $\mathbf{0}$ & 2.053 & 0.000 & $\mathbf{- 1 . 9 6 7}$ & 124 \\
\hline $\mathbf{1}$ & 2.045 & -0.070 & $\mathbf{- 1 . 9 7 5}$ & 124 \\
\hline 2 & 2.021 & -0.141 & $\mathbf{- 2 . 0 2 4}$ & 121 \\
\hline 3 & 1.976 & -0.211 & $\mathbf{- 2 . 0 6 8}$ & 118 \\
\hline 4 & 1.917 & -0.282 & $\mathbf{- 2 . 1 1 2}$ & 116 \\
\hline $\mathbf{5}$ & 1.846 & -0.352 & $\mathbf{- 2 . 2 1 0}$ & 111 \\
\hline $\mathbf{1 0}$ & 1.404 & -0.737 & $\mathbf{- 2 . 9 9 8}$ & 82 \\
\hline $\mathbf{1 5}$ & 1.419 & -0.902 & $\mathbf{- 0 . 4 7 4}$ & 517 \\
\hline $\mathbf{2 0}$ & 1.461 & -0.887 & 1.625 & 151 \\
\hline $\mathbf{2 5}$ & 1.237 & -0.995 & 0.000 & 662657 \\
\hline 30 & 0.947 & -1.203 & $\mathbf{- 1 . 4 3 1}$ & 171 \\
\hline 35 & 0.742 & -1.436 & $-\mathbf{1 . 9 2 7}$ & 127 \\
\hline $\mathbf{4 0}$ & 0.918 & -1.433 & 0.955 & 256 \\
\hline $\mathbf{4 5}$ & 1.274 & -1.235 & 3.540 & 69 \\
\hline $\mathbf{5 0}$ & 1.493 & -1.108 & 2.944 & 83 \\
\hline $\mathbf{5 5}$ & 1.661 & -0.997 & 2.940 & 83 \\
\hline $\mathbf{6 0}$ & 1.801 & -0.884 & 3.094 & 79 \\
\hline $\mathbf{6 5}$ & 1.920 & -0.759 & 3.354 & 73 \\
\hline $\mathbf{7 0}$ & 2.021 & -0.620 & 3.606 & 68 \\
\hline $\mathbf{7 5}$ & 2.099 & -0.474 & 3.780 & 65 \\
\hline $\mathbf{8 0}$ & 2.156 & -0.319 & 3.931 & 62 \\
\hline $\mathbf{8 5}$ & 2.191 & -0.160 & 4.012 & 61 \\
\hline $\mathbf{9 0}$ & 2.202 & 0.000 & 4.034 & 61 \\
\hline & & & & \\
\hline
\end{tabular}

\subsubsection{Vortex shedding}

Vortex shedding occurs if the vortices periodic break from the opposite edges of the structure and, as the result, variable load perpendicular to the direction of the wind occur.

If the frequency of the vortex excitations coincides with the natural frequency of the structure, large amplitude oscillations may arise. This occurs at the so-called critical wind speed:

$$
V_{c r, i}=\frac{b \cdot f_{i}}{S h}
$$

where:

b - reference width of the cross-section at which resonant vortex shedding occurs and where the modal deflection is maximum for the structure or structural part considered;

$f_{i}$ - natural frequency of the considered flexural mode i of cross-wind vibration;

Sh - Strouhal number. 
According to [4], the effect of vortex shedding should be investigated when the ratio of the largest to the smallest crosswind dimension of the structure, both taken in the plane perpendicular to the wind, exceeds 6 .

The effect of vortex shedding need not to be investigated when

$$
V_{c r, i}>1.25 \cdot V_{m}
$$

where:

$V_{c r, i}$ - is the critical wind velocity for mode $\mathrm{i}$;

$V_{m}$ - is the characteristic 10 minutes mean wind velocity.

Below there are the results of unsteady simulations, performed using the specialized software ANSYS CFX. The main frequencies of vortex shedding at various angles of attack were determined (Fig.4). Then, for two lowest (main) frequencies the Strouhal numbers $S h$ were calculated and the critical wind speed (at $f_{l}=0.4 \mathrm{~Hz}$ ) was determined using formula (5). For all angles of attack the critical wind speed did not exceed the value $1.25 \cdot V_{m}=27.1$ $\mathrm{m} / \mathrm{s}$; therefore, according to the Eurocode, vortex shedding can occur at the considered angles of the wind attack (table 3 ).
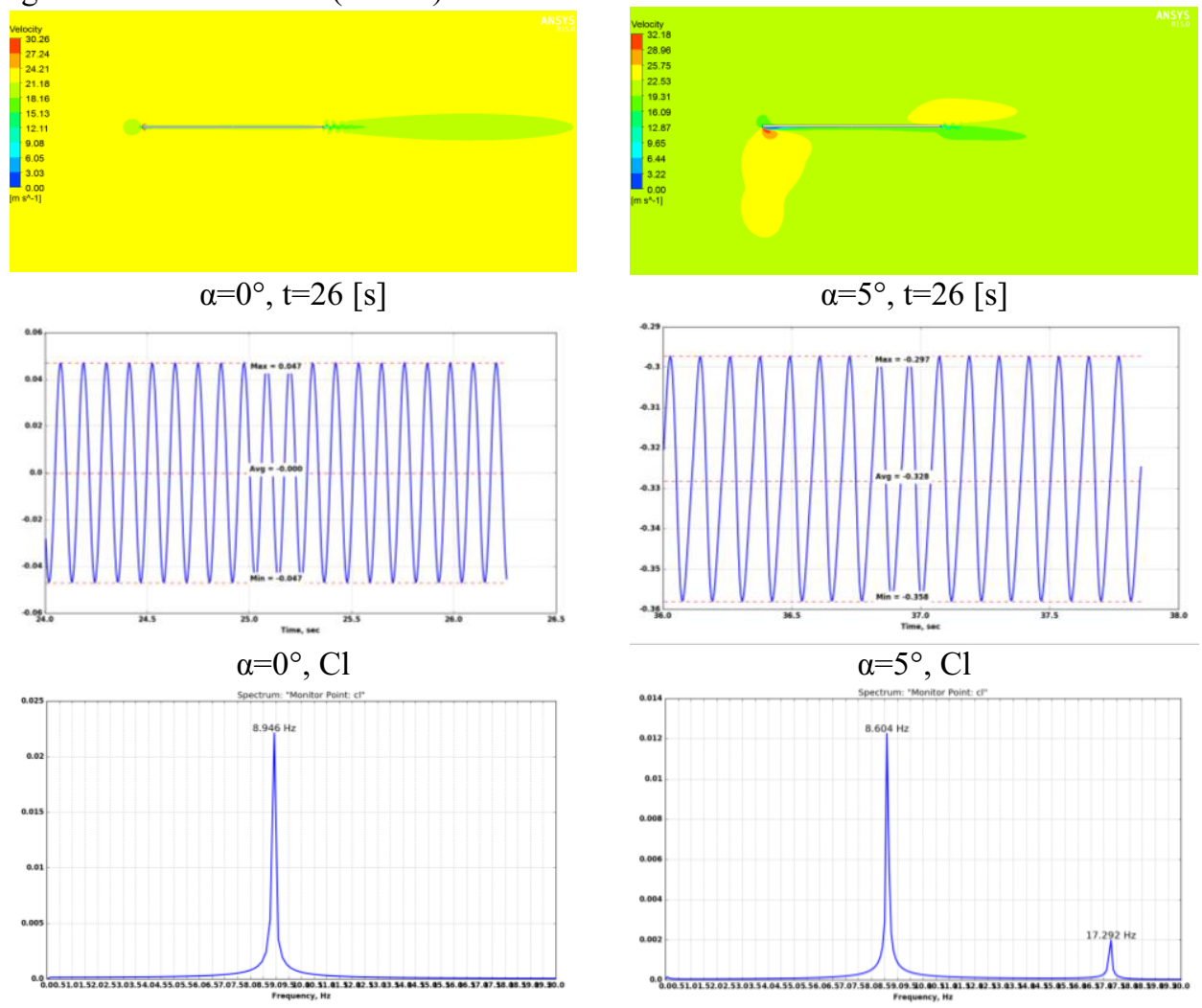

$\alpha=0^{\circ}, \mathrm{f}[\mathrm{Hz}]$

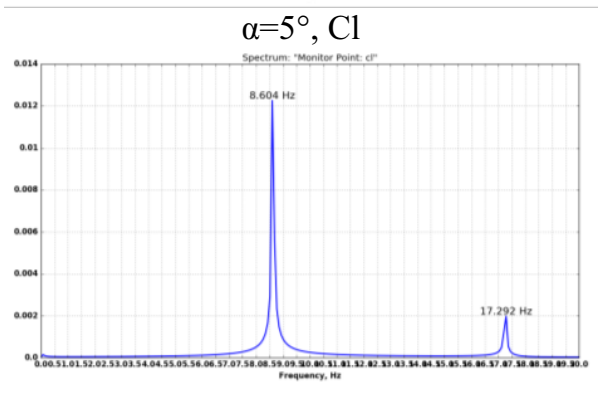

$$
\alpha=5^{\circ}, \mathrm{f}[\mathrm{Hz}]
$$

Fig.4. Results of the unsteady simulations (Model 4) for angel of attack $\alpha=0^{\circ}$ and $\alpha=5^{\circ}$ - from left to right. Field of instantaneous velocity $[\mathrm{m} / \mathrm{s}]$, aerodynamic coefficient of the lift force $(\mathrm{Cl})$, PSD for $\mathrm{Cl}$-from top to bottom. 
Table 3. Strouhal numbers $S h$ and critical wind velocity $V_{c r, 1}$ on angle of the wind attack $\alpha$.

\begin{tabular}{|c|c|c|c|c|}
\hline Angle $\boldsymbol{\alpha},^{\circ}$ & Sh1 & Sh2 & Ver,1(Sh1) & Ver,1(Sh2) \\
\hline 0 & 0.205 & - & $\mathbf{0 . 9 7}$ & - \\
\hline 1 & 0.204 & 0.407 & $\mathbf{0 . 9 8}$ & $\mathbf{0 . 4 9}$ \\
\hline 2 & 0.201 & 0.402 & $\mathbf{1 . 0 0}$ & $\mathbf{0 . 5 0}$ \\
\hline 5 & 0.200 & 0.399 & $\mathbf{1 . 0 0}$ & $\mathbf{0 . 5 0}$ \\
\hline
\end{tabular}

\subsubsection{Divergence and Flutter}

According to [4], such types of dynamic instability as divergence and flutter, which occur if structural deformations cause an aerodynamic loads changing, should be excluded in principle. To be prone to either divergence or flutter, the structure satisfies all of the three criteria specified in the Eurocode. If all the conditions are met, it is necessary to check the structure under study for the possibility of a flutter or divergence arising from the condition:

$$
V_{d i v}>2 \cdot V_{m}
$$

The critical wind velocity for divergence $V_{d i}$ is given in Expression (8):

$$
V_{d i v}=\left[\frac{2 \cdot k_{\Theta}}{\rho \cdot d^{2} \cdot \frac{d c_{M}}{d \Theta}}\right]^{\frac{1}{2}}
$$

where:

$k_{\Theta}$ - torsional stiffness; $c_{M}$ - aerodynamic moment coefficient; $\frac{d c_{M}}{d \Theta}$ - rate of change of aerodynamic moment coefficient with respect to rotation about the torsional centre, $\Theta$ is expressed in radians; $M$ - aerodynamic moment of a unit length of the structure;

$\rho$ - density of air; $\mathrm{d}$ - in wind depth (chord); b - width.

Results of the unsteady simulations are shown below. Values of the aerodynamic moment $\mathrm{Cmz}$ at different angles of attack were determined. Since all three conditions specified in the Eurocode are satisfied for the present structure, the critical wind speed for divergence was calculated (for $k_{\Theta}=14.23 \cdot 10^{4} \mathrm{Nm}^{2}, \rho=1.185 \mathrm{~kg} / \mathrm{m}^{3}$ ) for all angels of attack according to formula (8). In the table 4 , those angles for which the critical wind speed $V_{d i v}$ does not exceed $2 \cdot V_{m}=43.4 \mathrm{~m} / \mathrm{s}$ are marked in red. For these angles, when a corresponding wind speed is reached, a divergence may occur (Table 4 ).

Table 4. Aerodynamic moment coefficients $\mathrm{Cmz}$ and critical wind velocity $V_{d i v}$ on angle of the wind attack $\alpha$.

\begin{tabular}{|c|c|c|}
\hline Angle $\boldsymbol{\alpha}^{\circ}{ }^{\circ}$ & $\mathbf{C m z}$ & $V_{d \dot{v}}, \mathbf{M} / \mathbf{c}$ \\
\hline $\boldsymbol{0}$ & 0.000 & $\mathbf{1 0 . 3 9}$ \\
\hline 1 & 0.020 & $\mathbf{1 0 . 3 9}$ \\
\hline 2 & 0.040 & $\mathbf{1 0 . 4 3}$ \\
\hline 3 & 0.060 & $\mathbf{1 0 . 6 4}$ \\
\hline
\end{tabular}




\begin{tabular}{|c|c|c|}
\hline Angle $\boldsymbol{\alpha},{ }^{\circ}$ & $\mathbf{C m z}$ & $V_{d i v}, \mathbf{M} / \mathbf{c}$ \\
\hline 4 & 0.078 & $\mathbf{1 0 . 8 3}$ \\
\hline 5 & 0.097 & $\mathbf{1 0 . 8 4}$ \\
\hline $\mathbf{1 0}$ & 0.179 & $\mathbf{1 1 . 5 5}$ \\
\hline $\mathbf{1 5}$ & 0.140 & $\mathbf{1 6 . 8 5}$ \\
\hline $\mathbf{2 0}$ & 0.123 & $\mathbf{2 5 . 1 1}$ \\
\hline $\mathbf{2 5}$ & 0.123 & 360.18 \\
\hline $\mathbf{3 0}$ & 0.126 & 59.85 \\
\hline $\mathbf{3 5}$ & 0.127 & 91.26 \\
\hline $\mathbf{4 0}$ & 0.127 & -0.005 \\
\hline $\mathbf{4 5}$ & 0.124 & -0.030 \\
\hline $\mathbf{5 0}$ & 0.119 & -0.066 \\
\hline $\mathbf{5 5}$ & 0.110 & $\mathbf{- 0 . 0 9 8}$ \\
\hline $\mathbf{6 0}$ & 0.099 & $\mathbf{- 0 . 1 2 4}$ \\
\hline $\mathbf{6 5}$ & 0.086 & $\mathbf{- 0 . 1 4 9}$ \\
\hline $\mathbf{7 0}$ & 0.071 & $\mathbf{- 0 . 1 7 1}$ \\
\hline $\mathbf{7 5}$ & 0.055 & $\mathbf{- 0 . 1 8 7}$ \\
\hline $\mathbf{8 0}$ & 0.037 & $\mathbf{- 0 . 2 0 3}$ \\
\hline $\mathbf{8 5}$ & 0.019 & $\mathbf{- 0 . 2 1 1}$ \\
\hline $\mathbf{9 0}$ & 0.000 & $\mathbf{- 0 . 2 1 6}$ \\
\hline & & \\
\hline
\end{tabular}

\section{Conclusions}

Present paper is devoted to the computational investigations of aerodynamic instability of thin flat plate placed in a viscous incompressible turbulent flow. For this structure a check of the conditions for the occurrence of galloping, divergence and flutter was conducted. Obtained results can be used as a reference for the investigation of the sensitivity of structures like thin roofs of the large-span stadiums or bridges to wind loads.

\section{Acknowledgments}

The Reported study was funded by Government Program of the Russian Federation «Development of science and technology» (2013-2020) within Program of Fundamental Researches of Ministry of Construction, Housing and Utilities of the Russian Federation and Russian Academy of Architecture and Construction Sciences, the Research Project 7.1.2 and Ministry of Education and Science of the Russian Federation (RF President Grant, agreement \#14.Z56.16.8493-MK).

\section{References}

1. N. I. Afanasyeva, I. Yu. Lantsova, Numerical simulation of an elastic structure behavior under transient fluid flow excitation AIP Conference Proceedings 1800, Issue 1, (2017)

2. E. Simiu, R. Skanlan, Wind influence on buildings and structures. Moscow: Stroiizdat, (1984)

3. G.A. Savitsky Wind load on structures. Moscow: Publishing House of Literature on Construction (1972)

4. EN 199114:2005. Eurocode 1: Actions on structures - Part 14: General actions - Wind actions. Brussels: CEN TC 250, (2004) 